\title{
Enhance Flexibility in Human Agent Interaction a Case Study on IMAS Agent System
}

\author{
Xu Liang ${ }^{1,2}$, Peng Zhang ${ }^{2}$, Guohua Bai ${ }^{2}$ \\ ${ }^{1}$ School of Management and Economics, Kunming University of Science and \\ Technology, Kunming, Yunnan, 650093, China \\ ${ }^{2}$ School of Engineering, Blekinge Institute of Technology (BTH), \\ Box 520,S-372 25, Ronneby, Sweden \\ xlemkm@163.com,pengzhang80@hotmail.com,gba@bth.se
}

\begin{abstract}
Human Agent Interaction (HAI) is considered as a sub area in Human Computer Interaction (HCI), in which the computer systems are specified as agent systems. Computer agents are different from traditional Information Systems in aspects of autonomy, adaptivity, and persona when interacting with human users. The usability principles in HCI research should be refined to focus on agent special characteristics when designing agent systems. We argue that flexibility should be especially enhanced in such a case.
\end{abstract}

\section{Introduction}

Human Agent Interaction is considered as a sub area in Human Computer Interaction, in which the computer system is specified as agent system. The interpretation of a computer agent is different from that of a traditional Information System, which may help people to make rational decisions via commands and/or direct manipulations. A computer agent can collaborate with the user and is engaged in a co-operative process in which humans and agents both initiate communication, monitor events and perform tasks ${ }^{[1]}$ (Maes 1994).

The special characteristics of agent, i.e., autonomy, determine that the interaction between agent and human is different from other computer systems. The usability principles in human agent interactions should also be specified to focus on agent special characteristics.

In the rest of the paper, we will first briefly discuss the trend in traditional Human Computer Interaction (HCI) development. Secondly we argue that flexibility should be enhanced when designing the interaction between human users and computer agents. Then we compare the XMIS Web based system and agent based system in order to illustrate the flexibility in human agent interaction. In the end, we give a discussion and conclusion.

\section{Trend in human computer interaction}

Usability is a buzz word that has been used a lot when referring to the topic of Human Computer Interaction (HCI). Many researchers have presented their own understandings and research contributions in this area. Lauesen ${ }^{[2]}$ defined six factors of usability: fit for use, ease of learning, task efficiency, ease of remembering, subjective satisfaction, and understandability. Dix et al. ${ }^{[3]}$ introduced some principles to promote the usability of interactive systems.

Most of usability principles fall into three main categories, learnability, flexibility, and robustness. Learnability indicates the ease with which new users can begin effective interaction and achieve maximal performance. Flexibility indicates the multiplicity of ways the user and system exchange information. Robustness indicates the level of support provided to the user in determining successful achievement and assessment of goals.

The interaction between human users and computer system has evolved through three stages:

- Command line: A typical example is DOS. Users must memorize the commands in order to manipulating the computers. The commands have strict grammars, which must be followed by the users. Command line interfaces, even though offer direct access to system functionality, are hard to use. However, command line is still being used by some experienced users.

- Text based interface (menu, Question and Answer): for example, menu based interfaces 
offer users some choices in hierarchical menus. Users do not have to type in the commands, which makes the system easy to use. The weakness of text based interface is that the structure is designed before the run-time. For example, the structure of the menu is static once the system has been put into use.

- WIMP: WIMP (window, icon, menu, and pointer) is currently the most popular interface style, e.g., MS Windows. With WIMP, users have much freedom in choosing the way that they want the tasks to be conducted, even though many systems also offer wizards for users to quickly finish the tasks.

Clearly there is a trend in the development of the interaction between human users and computer systems towards offering more and more freedom and flexibility to human users in choosing how they wish to manipulate the computers.

We believe that this trend will continue and the new computing paradigm - agent - will very much influence this trend. The autonomy of agent systems indicates that agent systems can make plans and decisions on their own behalf. In this case, users do not have to bother with some miscellaneous information processes. And we believe that agent will dominate the computer systems design and bring a new topic to researchers - Human Agent Interaction (HAI)

\section{Human agent interaction: flexibility in focus}

We argue that flexibility in HAI should refer to not only the multiplicity of ways that users and system exchange information, but also the multiplicity of processes that users and system cooperate to conduct the tasks.

Software agents work to achieve predefined goals on behalf of their users. Using agents to solve real problems has become a new trend in many fields, e.g., health care ${ }^{[4]}$, port management ${ }^{[5]}$, Ecommerce ${ }^{[6]}$, traffic simulation ${ }^{[7]}$,information searching and filtering ${ }^{[8]}$ etc. Some unique characteristics of software agents should be manifested in their interactions with human users, namely, autonomy, adaptivity, and persona.

An intelligent agent is considered to be capable of autonomy, reactivity, proactivity, and social ability ${ }^{[9]}$. An agent can quickly response to the environments (reactivity), deliberates based on its internal states and general new goals (proactivity), and coordinate its activities with other agents (social activities).

Autonomy makes indirect manipulation possible for the users. Indirect manipulation means that the user does not need to bother with controlling the process that a computer system does the tasks.

To complete a task, corresponding knowledge and capabilities are necessary. An agent can hide the process of conducting a task from the users and release them from miscellaneous manipulations. In this case, agent systems are not only a system that listens to the commands from users, but also an autonomic entity that can positively interact with users.

Obviously more flexibility is required in HAI, compared with traditional HCI which presents detailed task process and direct manipulation to the users. Figure 1 illustrates that the direct manipulation present all task process details to the users who have to handle all the details of accomplishing the task. With indirect manipulation agents only ask the users to configure their preferences and the expected outcome of the tasks. The agents will handle the details of the task process and report the outcomes to the users later.

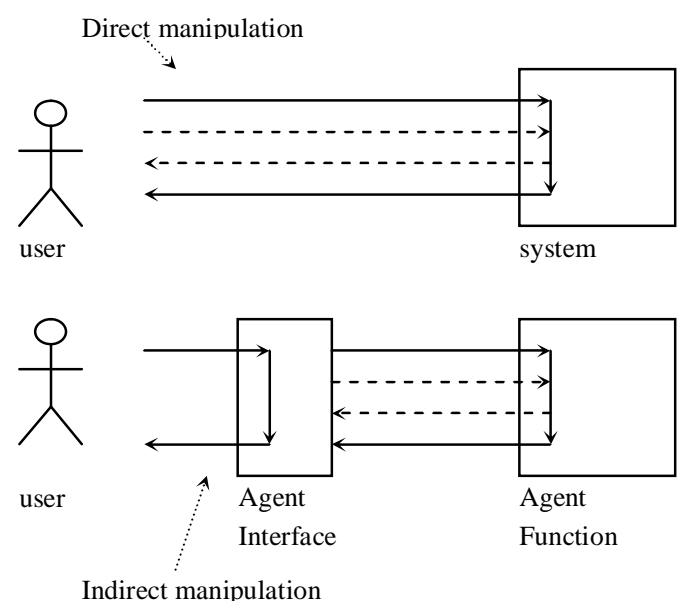

Figure 1. Indirect manipulation vs. direct manipulation

\subsection{Collaborative Interface}

To accomplish some complex tasks, multi-users are need to cooperate. For example all members are requested to join at the same time when they are making group decision. So the user interface must have collaborative to coordinate each user's operation. The communication function of multiagents system can provide the collaboration mechanism. 
The collaboration can be broken down into three layers, collaboration, coordination and communication. Collaboration happens among the users and support by Coordination and communication. Coordination and communication happen among interface agents. Each user interface is assisted with an agent.

The collaboration is the interaction activities of a group of relevant users. These activities can be defined through user needs specification, Such as voting management, calendar arrangement, task delegation and so on.

\subsection{Self-Adaptive Interface}

Agents can adapt itself interaction with users at runtime, when there are changes in the environment. The adaptive interaction can be user-initiated or agent-initiated, depending on to what extent the user is involved in the adaptations.

User-initiated adaptivity is called customization. In this case, the users take initiative to change the system in the way they want. Customization enables the users to manually choose the style and the functions that they prefer in the system.

Agent-initiated adaptivity is called personalization. In personalization, the user is seen as being more passive, while the system monitors, analyzes and reacts to the behavior of the user, although users can still configure some parameters ${ }^{[10]}$. Personalization enables the system to change the style and functions based on the users' previous manipulations without the notice to users. That is, the users do not need to do anything to make changes. Obviously, personalization is more difficult to implement than customization.

\subsection{Personas Interface}

Agents may represent persona in the reality. For example, an assistant agent may accept task delegations from their users. When performing these tasks, the agents are considered to have the same persona as the human users have in the reality.

The notion of agent prompts the computer system to a level on which it may interact equally with human users. The computer system is not a purely passive listener any longer. Besides, interactions among agents are also necessary and important. Since users are partially released from the burden of tasks execution, the agent should take their roles instead. When coordinating with other agents and users, the agent should reflect the role or persona from its human user.
Agent persona involves social aspects, e.g., social categorizations, norms and rules, which should be taken into account when modeling the interaction between users and agents. Katagiri et al ${ }^{[11]}$ argued that social factors such as affiliation, authority and conformity, need to be taken into account in interface agent design, as they can have effective and persuasive power in human-agent interaction

\section{Case study: XMAS agent interaction compared with XMIS Web based system}

In the research project XMIS for healthcare, we developed two systems for the diabetic healthcare actors. XMIS Web System, as the name indicates, is a Web based information system. The other one, XMAS is an agent based system that runs in Java compatible environments.

XMIS Web system was developed as a communication platform for diabetic healthcare users. So the users, both care providers and care receivers, share a common communication channel. The system provides messaging, calendar, diabetic related analysis tools etc. The system requires user login for authentication. Users are authorized with different roles, which are associated with different permissions and restrictions in using the XMIS system.

XMAS system is a further development to the XMIS Web systems. Based on the functionalities, the XMAS system was developed with Agent technology and aims to provide the actors with agent-based services, such as, real time glucose monitoring, alarm, calendar arrangement, task delegation etc.

The functionalities of the two systems are similar. However, their ways of interacting with users are very much different. Hereby we choose functionalities of calendar arrangement and glucose management as examples to compare the two systems.

\subsection{Calendar arrangement with collaborative manipulation}

Users may delegate meeting activity to the XMAS agents. Figure 2 illustrates that the user tells the agent necessary information about the meeting, such as, time, length, location, expected participants. Then the agent will automatically communicate with the agents of the expected participants. All agents negotiate with each other using the predefined 
protocol. After that, meeting alternatives will be present to the users, who will finally make the decision to choose one meeting and confirm it. Then the agents will put up the meeting activity to the calendars of their users.

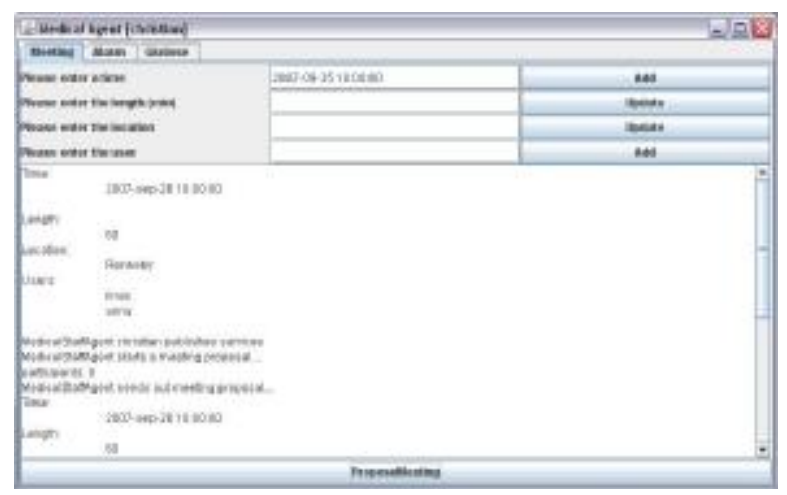

Figure 2 XMAS agent proposal a meeting

XMIS took a normal approach of negotiating a meeting. The users personally must contact each other via phone, emails or XMIS message service to check with each other about their calendars. After that they will put up the meeting activity in their calendar separately, as Figure 3 shows. The whole process takes a lot of time, especially when there are more than two participants.

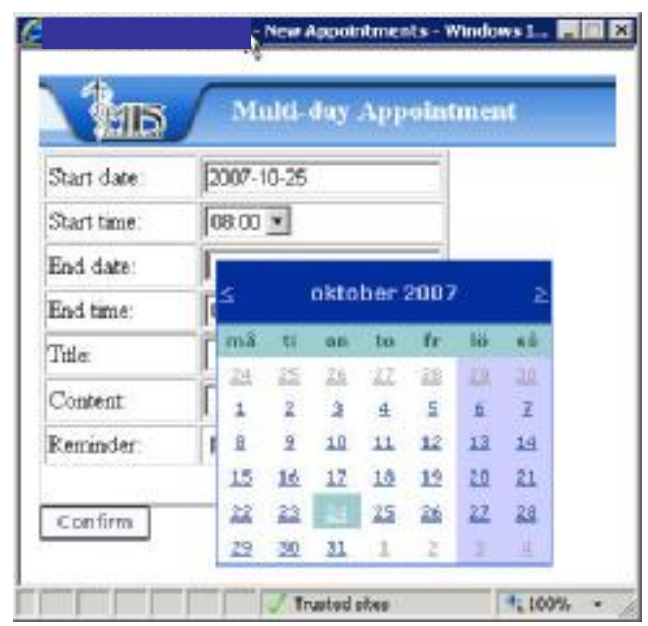

Figure 3 Actors may book an activity in their calendars

\subsection{Adaptive interaction}

There is a Knowledge Base component in individual architecture of XMAS agents. Knowledge Base stores knowledge and information that agents need to perform tasks. One important part of the knowledge is the Environment Model. Environment
Model defines the rules, labor division and community (other relevant agents), when the agent is performing a specific task. Take an example of the glucose management. XMAS Patient Agent monitors the glucose level of the patient. When it detects a possible acute situation, it will send alarms to relevant agents. The agents that will receive the alarm are chosen from the community based on the rule and labor divisions. Preferences in this selection are defined with factors such as, relations to the patient, the closest person to the patient at a specific time period and so on. Figure 4 shows that a parent receives an alarm that tells the glucose level of her boy Linus is rather high at this moment. The alarm is sent to her since it is around noon on Sunday, so the boy should be at home or other places that the parent should be able to reach quickly. In other occasions, the alarm might be sent to the school nurse, if the boy is supposed to be in school.

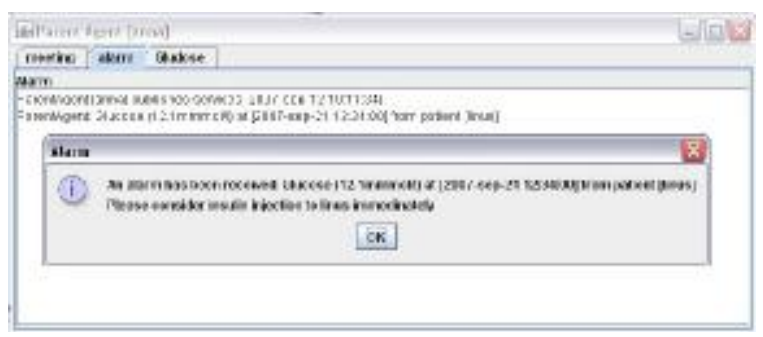

Figure 4 Patient Agent decides to send the alarm to the parent by processing its Knowledge Base.

XMIS Web system requires the user to manually choose one or more care providers to write messages, which can be about anything regarding the health status of him/her. See Figure 5.

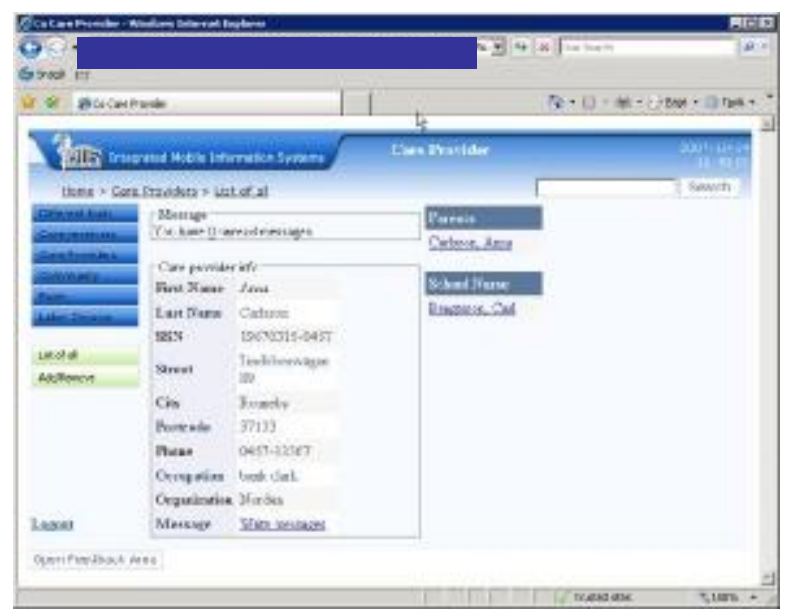

Figure 5 Patient has to manually choose a care provider to send messages. 


\subsection{Persona reflection}

The persona of XMAS agent is manifested in twofold. On one hand, the agent is able to represent the persona from its human users. The persona reflects to some extent the role of its users in the reality. For example, the when an agent starts a meeting proposal, it represents a role in the reality, e.g., doctor. Associated with the role, the agent is authorized to be permitted to call up such a meeting.

On the other hand, the agent is able to understand the persona of other agents. That is, the agent knows about the identities of other agents. This is realized with the Knowledge Base that is built in the agent architecture. For example, the agent is able to adaptively choose relevant agents to send alarms or other messages.

\section{Discussion}

A successful design of the interaction between users and computer systems involves many issues. Flexibility is only one important aspect when considering the interaction with agent systems. Still there are other issues that are necessary, e.g., learnability and robustness, trust, security/privacy etc.

- Learnability and robustness: other usability issues, i.e., learnability and robustness, are still valid and should be seriously considered.

- Trust: it is important for users to trust a system which will directly interact with humans. Agents may be applied to perform a simulation of human activities, or perform real world tasks for human users. In both cases, how to help users trust the agent is both a social issue and a technical one in the case how to design the interaction between human and agents.

- Security/privacy: The introduction of computer agents brings improved usability together with some new threats. When delegating tasks to computer agents, human users should be confirmed that the security and privacy issues, which was under the control of human beings before, are now correctly and safely handled by the computer agents

\section{Conclusion}

We argue that in a specific area of HCI, i.e., HAI, more concentration should be put on the flexibility. The intrinsic characteristics of computer agents determine that agents should provide human users with automatic and adaptive services, and reflecting the social status of their human users.

Thus, a refinement of the view on usability is recommended when considering HAI. The autonomy of agents suggests that the indirect manipulation should be manifested via agent interface. Customization and personalization in agent interface are recommended to improve the adaptive interaction between agents and human users. As an intelligent and 'human-like' entity, agent also possesses social status in a specified settings, thus we recommend that agent persona and other social aspects should also be manifested in HAI.

\section{References}

[1] Maes, P. (1994). "Agents That Reduce Work and Information Overload." Communications of the ACM 37(7).

[2] Lauesen, S. (2005). User Interface Design - a software engineering perspective, Addison Wesley.

[3] Dix, A., J. Finlay, et al. (2004). Human-Computer Interaction, Pearson Prentice Hall.

[4] Nealon, J. L. and A. Moreno (2003). Agent-Based Applications in Health Care. Applications of Software Agent Technology in the Health Care Domain. Basel, Germany: 3-18.

[5] Henesey, L., T. Notteboom, et al. (2003). Agent-based simulation of stakeholders relations: An approach to sustainable port and terminal management. International Association of Maritime Economists Annual Conference, Busan, Korea.

[6] Arafa, Y. and A. Mamdani (2001). Building Multimodal Personal Sales Agents as Interfaces to Ecommerce Applications.

[7] Bazzan, A. L. C. (2005). "A Distributed Approach for Coordination of Traffic Signal Agents." Autonomous Agents and Multi-Agent Systems(10): 131-164.

[8] FEI Hong-xiao, GONG Yan-ling etc , 2006, "MultiAgent system for information filtering based on hybrid learning approach" Journal of Computer Applications ,2006.02

[9] Wooldridge, M. (2001). An Introduction to Multiagent Systems, JOHN Wiley \& Sons Ltd.

[10] Schiaffino, S. and A. a. Amandi (2005). "An Interface Agent Approach to Personalize Users' Interaction with Databases." Journal of Intelligent Information Systems 25(3): 251-273.

[11] Katagiri, Y., T. Takahashi, et al. (2001). Social Persuasion in Human-Agent Interaction. Second IJCAI Workshop on Knowledge and Reasoning in Practical Dialogue Systems, Seattle. 